this atlas would be vastly improved by pairing drawings with matching per-operative pictures, but there are obvious financial and medicolegal (this is an American publication) constraints to this. I paid particular attention to the surgical approaches involving the orbit (transconjunctival + lateral canthotomy, subciliary, infraorbital, brow) and found the layout and detail a useful résumé for the already practising surgeon who knows what needs to be achieved but wants a reminder of the options; the content is therefore insufficient for the novice.

Over half the atlas is devoted to the management of mandibular problems and as such is of no purchase interest to an ophthalmic surgeon - even one with orbital interest - nor to an Eye Unit library.

Michelle Beaconsfield

\section{Ocular Cytopathology}

B. J. Glasgow and R. Y. Foos

Butterworth-Heinemann, Boston, 1993.

This is a beautifully and copiously illustrated book. This is a mixed blessing. One hundred and ninetythree photographs are included in its one hundred and twenty-eight pages and each of the ten chapters of pithy text is well referenced. The techniques involved in harvesting cells for cytological evaluation are described in chapter 1 , but whilst reading this section it seemed inappropriate to me to include photographs of a cotton wool bud and an Ependorf microfuge tube. This unease prompted me to examine just how many of these handsome images were relevant and how many were cytological preparations rather than histological or clinical.

The tally was 9 of the 23 in the chapter on normal anatomy and cytological features, 8 (including 3 Giemsa stains) of the 16 illustrations to the external diseases chapter, and only 3 of the 18 on the iris ciliary body and angle. In the vitreous, however, the score rises to 6 of 11 (although 2 of these were the same field with and without polarised light), and by the time we reach the retina and the diagnosis of endophthalmitis the score starts to rise with 13 out of 21. The chapter on the intraocular neoplasms was good, with 10 cytological illustrations, but when it came to the orbit only 20 of the 51 were cytology, 2 were gross pathology, there were 13 illustrations of CT scans, 6 clinical pictures, 2 electron micrographs and 6 histological sections. In fact, well under half of the illustrations in the book were from cytological material. Why?

The answer is simple enough. The authors, as with all of us who do ocular cytopathology, are 'learning on the job'. Fine needle aspiration cytology of the eye and adnexae is a relatively new technique, although paracentesis of the anterior chamber is not. Smears from the conjunctiva and cornea may fall into the microbiologist's hands and most orbital surgeons seek reassurance from histological examination and diagnosis except in the case of accessible metastatic lesions in the orbit of frail patients. There has therefore been a dearth of material, coupled with a certain reluctance to believe the results obtained from a few cells. This is changing as more cytopathologists are willing to look at ocular material and ophthalmic pathologists are learning the harvesting and diagnostic techniques used by their colleagues.

The laboratory at the Jules Stein has always been refreshingly innovative whilst at the same time having a long tradition of good ophthalmic pathology rooted in histological examination. Less invasive techniques are increasingly being used in surgery and it is logical to wish that cellular diagnostic techniques should also become minimalist. This aim cannot be realised unless the surgeon or oncologist has confidence in the results. The comparison of histological and cytological material is relevant and use of this book should enable pathologists to feel more confident in handling small quantities of material. I am sure that subsequent editions will show an increasing ratio of cytological illustrations. Ophthalmologists, especially those taking the final Fellowship, can expect to use the text as a refreshing revision that is undoubtedly 'easy on the eye' and may provide a visual aide mémoire.

Alison McCartney

\section{Neuro-Ophthalmological Disorders: Diagnostic Work-up and Management}

\section{R. J. Tusa and S. A. Newman}

Marcel Dekker, New York, 1994.

This book is the latest volume in the series 'Neurological Disease and Therapy'. Aimed at neurologists, neurosurgeons and ophthalmologists there are 33 volumes in the series so far. The present volume has 54 contributors to its 41 chapters, which are grouped into Afferent System, Efferent System, Cavernous Sinus/Orbit, Technological Advances in Neuro-Ophthalmology, and Pathology.

The aim of creating a practical text-book relevant to daily practice is achieved by most contributors. Many chapters, however, have few diagrams and all illustrations are in black and white. The bibliography is extensive in most chapters.

The book will appeal to neuro-ophthalmologists who may have other volumes in the series, but it is unlikely to be attractive to the general ophthalmologist looking for a core text in neuro-ophthalmology.

A. G. Tyers 